J. Phys. Chem. B, 2006, $\underline{x x x}, x x x-x x x$

SUPPORTING INFORMATION

For

\title{
Molecular Ordering and Structure of Quasi-spherical Solutes by Liquid Crystal NMR and Monte Carlo Simulations: the Case of Norbornadiene
}

\author{
C. Aroulanda ${ }^{\dagger,}$, C. Celebre ${ }^{*}$, G. De Luca ${ }^{\star}$ and M. Longeri ${ }^{*}$ \\ *XNMR SCAn group, Dipartimento di Chimica, Università della Calabria, 87036 \\ Arcavacata di Rende, Rende (Cs), Italy. \\ ${ }^{\dagger}$ Méthodologie RMN, UMR CNRS-UHP 7565, Université Henri Poincaré, BP 239, 54506 \\ Vandoeuvre-lès-Nancy cedex, France.
}


TABLE SI-1 : NBD's geometries in cartesian coordinates (see PAS framework from the Figure 1). The coordinates of $\mathrm{H}_{1}, \mathrm{H}_{3}$ and $\mathrm{H}_{7}$ are reported, the remaining proton coordinates being related by the $\mathrm{C}_{2 \mathrm{v}^{-}}$ symmetry of the solute (the references are reported in the main body of the work).

\begin{tabular}{|c|c|c|c|c|c|c|c|c|c|c|}
\hline & $\mathbf{T}_{\text {red }}$ & $\mathbf{x}_{1} / \AA$ & $\mathbf{y}_{1} / \AA$ & $z_{1} / \AA$ & $\mathbf{x}_{3} / \AA$ & $\mathbf{y}_{3} / \AA$ & $\mathbf{z}_{\mathbf{3}} / \AA$ & $\mathbf{x}_{7} / \AA$ & $\mathbf{y}_{7} / \AA$ & $\mathbf{z}_{7} / \AA$ \\
\hline From Ref. [8] & & 1.913 & 1.354 & 0.000 & 0.000 & -2.192 & 1.613 & 0.910 & 0.000 & 3.017 \\
\hline $\begin{array}{c}\text { From Ref. [33] } \\
\text { (in EBBA) }\end{array}$ & & 1.908 & 1.347 & 0.000 & 0.000 & -2.192 & 1.605 & 0.908 & 0.000 & 3.041 \\
\hline $\begin{array}{c}\text { From Ref. [33] } \\
\text { (in Phase IV) }\end{array}$ & & 1.853 & 1.312 & 0.000 & 0.000 & -2.192 & 1.549 & 0.876 & 0.000 & 2.925 \\
\hline RHF/6-31G** & & 1.932 & 1.320 & 0.000 & 0.000 & -2.142 & 1.613 & 0.890 & 0.000 & 2.975 \\
\hline $\begin{array}{c}\text { LSDA } \\
6-311++G^{* *}\end{array}$ & & 1.915 & 1.338 & 0.000 & 0.000 & -2.155 & 1.649 & 0.909 & 0.000 & 2.987 \\
\hline $\begin{array}{c}\text { B3LYP } \\
6-31++G^{* *}\end{array}$ & & 1.936 & 1.339 & 0.000 & 0.000 & -2.161 & 1.633 & 0.900 & 0.000 & 3.000 \\
\hline \multirow{8}{*}{$\begin{array}{l}\text { Optimised in } \\
\qquad \text { EBBA }^{a}\end{array}$} & 0.91 & 1.916 & 1.351 & 0.000 & 0.000 & -2.175 & 1.620 & 0.910 & 0.000 & 3.017 \\
\hline & 0.92 & 1.916 & 1.350 & 0.000 & 0.000 & -2.172 & 1.620 & 0.910 & 0.000 & 3.017 \\
\hline & 0.93 & 1.917 & 1.350 & 0.000 & 0.000 & -2.174 & 1.620 & 0.910 & 0.000 & 3.017 \\
\hline & 0.94 & 1.917 & 1.350 & 0.000 & 0.000 & -2.173 & 1.620 & 0.910 & 0.000 & 3.017 \\
\hline & 0.95 & 1.917 & 1.350 & 0.000 & 0.000 & -2.174 & 1.619 & 0.910 & 0.000 & 3.017 \\
\hline & 0.96 & 1.917 & 1.351 & 0.000 & 0.000 & -2.177 & 1.618 & 0.910 & 0.000 & 3.017 \\
\hline & 0.97 & 1.917 & 1.351 & 0.000 & 0.000 & -2.174 & 1.620 & 0.910 & 0.000 & 3.017 \\
\hline & 0.98 & 1.932 & 1.339 & 0.000 & 0.000 & -2.178 & 1.619 & 0.910 & 0.000 & 3.017 \\
\hline \multirow{8}{*}{$\begin{array}{l}\text { Optimised in } \\
\mathbf{M M}^{a}\end{array}$} & 0.91 & 1.932 & 1.342 & 0.000 & 0.000 & -2.196 & 1.639 & 0.910 & 0.000 & 3.017 \\
\hline & 0.92 & 1.932 & 1.342 & 0.000 & 0.000 & -2.195 & 1.639 & 0.910 & 0.000 & 3.017 \\
\hline & 0.93 & 1.933 & 1.342 & 0.000 & 0.000 & -2.197 & 1.638 & 0.910 & 0.000 & 3.017 \\
\hline & 0.94 & 1.937 & 1.343 & 0.000 & 0.000 & -2.187 & 1.652 & 0.910 & 0.000 & 3.017 \\
\hline & 0.95 & 1.932 & 1.343 & 0.000 & 0.000 & -2.199 & 1.637 & 0.910 & 0.000 & 3.017 \\
\hline & 0.96 & 1.932 & 1.340 & 0.000 & 0.000 & -2.198 & 1.641 & 0.910 & 0.000 & 3.017 \\
\hline & 0.97 & 1.932 & 1.337 & 0.000 & 0.000 & -2.198 & 1.639 & 0.910 & 0.000 & 3.017 \\
\hline & 0.98 & 1.931 & 1.344 & 0.000 & 0.000 & -2.201 & 1.635 & 0.910 & 0.000 & 3.017 \\
\hline \multirow{8}{*}{ 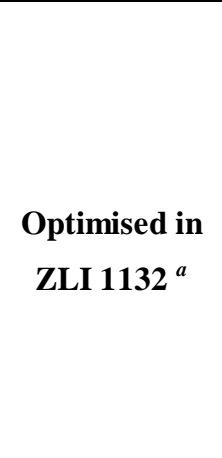 } & 0.90 & 1.942 & 1.345 & 0.000 & 0.000 & -2.175 & 1.643 & 0.910 & 0.000 & 3.017 \\
\hline & 0.91 & 1.941 & 1.345 & 0.000 & 0.000 & -2.178 & 1.642 & 0.910 & 0.000 & 3.017 \\
\hline & 0.92 & 1.941 & 1.344 & 0.000 & 0.000 & -2.177 & 1.643 & 0.910 & 0.000 & 3.017 \\
\hline & 0.93 & 1.942 & 1.345 & 0.000 & 0.000 & -2.177 & 1.642 & 0.910 & 0.000 & 3.017 \\
\hline & 0.94 & 1.941 & 1.345 & 0.000 & 0.000 & -2.178 & 1.642 & 0.910 & 0.000 & 3.017 \\
\hline & 0.95 & 1.941 & 1.345 & 0.000 & 0.000 & -2.179 & 1.641 & 0.910 & 0.000 & 3.017 \\
\hline & 0.96 & 1.941 & 1.344 & 0.000 & 0.000 & -2.177 & 1.643 & 0.910 & 0.000 & 3.017 \\
\hline & 0.97 & 1.942 & 1.345 & 0.000 & 0.000 & -2.179 & 1.642 & 0.910 & 0.000 & 3.017 \\
\hline
\end{tabular}

${ }^{a}$ Optimised in this work, leaving $\mathrm{H}_{7}$ fixed. 
TABLE SI-2 : "Equilibrium", Calculated and Observed Dipolar Couplings; Harmonic Vibrational Corrections, Deviations, Relative Corrections and $R$ for NBD at different temperatures in EBBA. Geometry and Force Field from B3LYP/6-31++G** calculations; experimental frequencies have been used in evaluating vibrational corrections (see text for more explanations). All dimensional values in $\mathrm{Hz}$.

\begin{tabular}{|c|c|c|c|c|c|c|c|}
\hline $\mathbf{T}_{\text {red }}$ & & $D_{i j}^{c e q} a$ & $D_{i j}^{h} b$ & $D_{i j}^{c a l c}{ }_{c}$ & $D_{i j}^{o b s} d$ & $\Delta^{e}$ & $\operatorname{Corr}^{f}$ \\
\hline \multirow{11}{*}{0.91} & $\mathrm{D}_{12}$ & 62.588 & -0.3023 & 62.2858 & 62.1030 & 0.1827 & -0.0049 \\
\hline & $\mathbf{D}_{13}$ & 4.978 & -0.0115 & 4.9667 & 5.5520 & -0.5853 & -0.0021 \\
\hline & $D_{14}$ & -20.368 & 0.1095 & -20.2583 & -20.2170 & -0.0413 & -0.0054 \\
\hline & $\mathbf{D}_{15}$ & -64.001 & -0.0662 & -64.0671 & -65.8900 & 1.8229 & 0.0010 \\
\hline & $D_{16}$ & -47.907 & -0.0653 & -47.9719 & -48.8160 & 0.8441 & 0.0013 \\
\hline & $\mathbf{D}_{17}^{b}$ & 42.874 & -0.1130 & 42.7613 & 42.9100 & -0.1487 & -0.0026 \\
\hline & $\mathbf{D}_{18}^{b}$ & -3.316 & -0.0580 & -3.3742 & -2.8520 & -0.5222 & 0.0203 \\
\hline & $D_{36}$ & 14.872 & -0.0647 & 14.8072 & 15.6260 & -0.8188 & -0.0041 \\
\hline & $\mathbf{D}_{37}$ & 49.844 & -0.4122 & 49.4322 & 48.9330 & 0.4992 & -0.0084 \\
\hline & $\mathbf{D}_{78}$ & -637.128 & 20.5978 & -616.5302 & -616.0200 & -0.5102 & -0.0334 \\
\hline & $\mathbf{R}$ & \multicolumn{6}{|c|}{0.71} \\
\hline \multirow{12}{*}{0.92} & & $D_{i j}^{c e q} a$ & $D_{i j}^{h} b$ & $D_{i j}^{c a l c} c$ & $D_{i j}^{o b s} d$ & $\Delta^{e}$ & $\operatorname{Corr}^{f}$ \\
\hline & $D_{12}$ & 59.836 & -0.2868 & 59.5496 & 59.3460 & 0.2036 & -0.0048 \\
\hline & $D_{13}$ & 4.733 & -0.0106 & 4.7223 & 5.1740 & -0.4517 & -0.0020 \\
\hline & $D_{14}$ & -19.710 & 0.1057 & -19.6042 & -19.6410 & 0.0368 & -0.0054 \\
\hline & $\mathrm{D}_{15}$ & -61.818 & -0.0636 & -61.8818 & -63.7440 & 1.8622 & 0.0010 \\
\hline & $D_{16}$ & -46.097 & -0.0619 & -46.1586 & -47.0060 & 0.8474 & 0.0013 \\
\hline & $\mathbf{D}_{17}^{b}$ & 41.588 & -0.1099 & 41.4782 & 41.6350 & -0.1568 & -0.0026 \\
\hline & $\mathbf{D}_{18}^{b}$ & -3.148 & -0.0564 & -3.2040 & -2.8110 & -0.3930 & 0.0201 \\
\hline & $\mathbf{D}_{36}$ & 14.218 & -0.0619 & 14.1561 & 14.9880 & -0.8319 & -0.0041 \\
\hline & $\mathbf{D}_{37}$ & 47.918 & -0.3987 & 47.5192 & 47.0690 & 0.4502 & -0.0085 \\
\hline & $\mathbf{D}_{78}$ & -615.398 & 19.8970 & -595.5010 & -594.9640 & -0.5370 & -0.0334 \\
\hline & $\mathbf{R}$ & \multicolumn{6}{|c|}{0.69} \\
\hline \multirow{12}{*}{0.93} & & $D_{i j}^{c e q}{ }_{a}$ & $D_{i j}^{h} \boldsymbol{b}$ & $D_{i j}^{\text {calc }}{ }_{c}$ & $D_{i j d}^{o b s}$ & $\Delta^{e}$ & $\operatorname{Corr}^{f}$ \\
\hline & $\mathbf{D}_{12}$ & 57.175 & -0.2721 & 56.9026 & 56.7780 & 0.1246 & -0.0048 \\
\hline & $\mathbf{D}_{13}$ & 4.500 & -0.0098 & 4.4901 & 4.9620 & -0.4719 & -0.0020 \\
\hline & $D_{14}$ & -19.036 & 0.1019 & -18.9337 & -18.9220 & -0.0117 & -0.0054 \\
\hline & $D_{15}$ & -59.606 & -0.0611 & -59.6674 & -61.3010 & 1.6336 & 0.0010 \\
\hline & $D_{16}$ & -44.299 & -0.0587 & -44.3575 & -45.1040 & 0.7465 & 0.0013 \\
\hline & $\mathbf{D}_{17}^{b}$ & 40.249 & -0.1066 & 40.1420 & 40.3240 & -0.1820 & -0.0026 \\
\hline & $\mathbf{D}_{18}^{b}$ & -2.988 & -0.0547 & -3.0429 & -2.6620 & -0.3809 & 0.0206 \\
\hline & $\mathbf{D}_{36}$ & 13.586 & -0.0592 & 13.5264 & 14.3420 & -0.8156 & -0.0041 \\
\hline & $\mathbf{D}_{37}$ & 46.012 & -0.3849 & 45.6273 & 45.1580 & 0.4693 & -0.0085 \\
\hline & $\mathbf{D}_{78}$ & -593.379 & 19.1865 & -574.1922 & -573.7200 & -0.4722 & -0.0334 \\
\hline & $\mathbf{R}$ & \multicolumn{6}{|c|}{0.62} \\
\hline \multirow{12}{*}{0.94} & & $D_{i j}^{c e q}{ }_{a}$ & $D_{i j}^{h} b$ & $D_{i j}^{c a l c}{ }_{c}$ & $D_{i j d}^{o b s}$ & $\Delta^{e}$ & $\operatorname{Corr}^{f}$ \\
\hline & $\mathbf{D}_{12}$ & 54.229 & -0.2557 & 53.9729 & 53.7920 & 0.1809 & -0.0048 \\
\hline & $\mathbf{D}_{13}$ & 4.241 & -0.0089 & 4.2317 & 4.6360 & -0.4043 & -0.0019 \\
\hline & $D_{14}$ & -18.302 & 0.0977 & -18.2040 & -18.2930 & 0.0890 & -0.0053 \\
\hline & $D_{15}$ & -57.191 & -0.0584 & -57.2495 & -58.8800 & 1.6305 & 0.0010 \\
\hline & $D_{16}$ & -42.324 & -0.0551 & -42.3795 & -43.1500 & 0.7705 & 0.0013 \\
\hline & $\mathbf{D}_{\mathbf{1 7}}^{b}$ & 38.797 & -0.1031 & 38.6942 & 38.8390 & -0.1448 & -0.0027 \\
\hline & $\mathbf{D}_{18}^{b}$ & -2.811 & -0.0529 & -2.8635 & -2.5330 & -0.3305 & 0.0209 \\
\hline & $D_{36}$ & 12.885 & -0.0561 & 12.8294 & 13.5650 & -0.7356 & -0.0041 \\
\hline & $\mathbf{D}_{37}$ & 43.917 & -0.3699 & 43.5468 & 43.1370 & 0.4098 & -0.0086 \\
\hline & $\mathbf{D}_{78}$ & -569.336 & 18.4107 & -550.9250 & -550.4450 & -0.4800 & -0.0334 \\
\hline & $\mathbf{R}$ & \multicolumn{6}{|c|}{0.61} \\
\hline \multirow[t]{3}{*}{0.95} & & $D_{i j}^{c e q}{ }_{a}$ & $D_{i j}^{h} b$ & $D_{i j}^{\text {calc }} c$ & $D_{i j}^{o b s} d$ & $\Delta^{e}$ & $\operatorname{Corr}^{f}$ \\
\hline & $D_{12}$ & 51.050 & -0.2386 & 50.8115 & 50.6300 & 0.1815 & -0.0047 \\
\hline & $D_{13}$ & 3.968 & -0.0081 & 3.9597 & 4.3310 & -0.3713 & -0.0019 \\
\hline
\end{tabular}


J. Phys. Chem. B, 2006, $x x x, x x x-x x x$

\begin{tabular}{|c|c|c|c|c|c|c|c|}
\hline & $\mathrm{D}_{14}$ & -17.448 & 0.0930 & -17.3555 & -17.4210 & 0.0655 & -0.0053 \\
\hline & $D_{15}$ & -54.422 & -0.0553 & -54.4771 & -55.9950 & 1.5179 & 0.0010 \\
\hline & $D_{16}$ & -40.117 & -0.0513 & -40.1688 & -40.9480 & 0.7792 & 0.0013 \\
\hline & $\mathbf{D}_{17}{ }^{b}$ & 37.076 & -0.0988 & 36.9776 & 37.1120 & -0.1344 & -0.0027 \\
\hline & $\mathbf{D}_{\mathbf{1 8}}{ }^{b}$ & -2.625 & -0.0507 & -2.6755 & -2.3570 & -0.3185 & 0.0215 \\
\hline & $D_{36}$ & 12.130 & -0.0529 & 12.0774 & 12.8310 & -0.7536 & -0.0041 \\
\hline & $\mathbf{D}_{37}$ & 41.587 & -0.3525 & 41.2348 & 40.8140 & 0.4208 & -0.0086 \\
\hline & $\mathbf{D}_{78}$ & -541.768 & 17.5208 & -524.2470 & -523.7960 & -0.4510 & -0.0334 \\
\hline & $\mathbf{R}$ & \multicolumn{6}{|c|}{0.59} \\
\hline \multirow{12}{*}{0.96} & & $D_{i j}^{c e q} a$ & $D_{i j}^{h} b$ & $D_{i j}^{c a l c}{ }_{c}$ & $D_{i j}^{o b s} d$ & $\Delta^{e}$ & $\operatorname{Corr}^{f}$ \\
\hline & $D_{12}$ & 47.571 & -0.2201 & 47.3507 & 47.1900 & 0.1607 & -0.0047 \\
\hline & $\mathbf{D}_{13}$ & 3.671 & -0.0071 & 3.6641 & 4.0310 & -0.3669 & -0.0018 \\
\hline & $D_{14}$ & -16.494 & 0.0877 & -16.4068 & -16.3620 & -0.0448 & -0.0054 \\
\hline & $D_{15}$ & -51.338 & -0.0520 & -51.3895 & -52.8080 & 1.4185 & 0.0010 \\
\hline & $D_{16}$ & -37.677 & -0.0473 & -37.7241 & -38.4910 & 0.7669 & 0.0012 \\
\hline & $\mathbf{D}_{\mathbf{1 7}}^{b}$ & 35.142 & -0.0939 & 35.0485 & 35.1670 & -0.1185 & -0.0027 \\
\hline & $\mathbf{D}_{\mathbf{1 8}}{ }^{b}$ & -2.423 & -0.0482 & -2.4716 & -2.1500 & -0.3216 & 0.0224 \\
\hline & $\mathbf{D}_{36}$ & 11.303 & -0.0493 & 11.2542 & 11.9640 & -0.7098 & -0.0041 \\
\hline & $\mathbf{D}_{37}$ & 39.015 & -0.3331 & 38.6822 & 38.2310 & 0.4512 & -0.0087 \\
\hline & $\mathbf{D}_{78}$ & -511.064 & 16.5294 & -494.5351 & -494.1270 & -0.4081 & -0.0335 \\
\hline & $\mathbf{R}$ & \multicolumn{6}{|c|}{0.50} \\
\hline \multirow{12}{*}{0.97} & & $D_{i j}^{c e q} a$ & $D_{i j}^{h} b$ & $D_{i j}^{c a l c}{ }_{c}$ & $D_{i j}^{o b s} d$ & $\Delta^{e}$ & $\operatorname{Corr}^{f}$ \\
\hline & $\mathbf{D}_{12}$ & 43.648 & -0.1997 & 43.4485 & 43.2440 & 0.2045 & -0.0046 \\
\hline & $\mathbf{D}_{13}$ & 3.343 & -0.0062 & 3.3369 & 3.6760 & -0.3391 & -0.0017 \\
\hline & $D_{14}$ & -15.364 & 0.0815 & -15.2821 & -15.2950 & 0.0129 & -0.0053 \\
\hline & $\mathbf{D}_{15}$ & -47.713 & -0.0481 & -47.7612 & -49.0680 & 1.3068 & 0.0010 \\
\hline & $D_{16}$ & -34.856 & -0.0429 & -34.8988 & -35.5690 & 0.6702 & 0.0012 \\
\hline & $\mathbf{D}_{17}^{b}$ & 32.822 & -0.0880 & 32.7342 & 32.8310 & -0.0968 & -0.0027 \\
\hline & $\mathbf{D}_{\mathbf{1 8}}{ }^{b}$ & -2.202 & -0.0452 & -2.2468 & -1.9970 & -0.2498 & 0.0226 \\
\hline & $D_{36}$ & 10.371 & -0.0453 & 10.3262 & 11.0330 & -0.7068 & -0.0041 \\
\hline & $\mathbf{D}_{37}$ & 36.054 & -0.3101 & 35.7436 & 35.4070 & 0.3366 & -0.0088 \\
\hline & $\mathbf{D}_{78}$ & -474.983 & 15.3639 & -459.6190 & -459.2380 & -0.3810 & -0.0335 \\
\hline & $\mathbf{R}$ & \multicolumn{6}{|c|}{0.50} \\
\hline \multirow{12}{*}{0.98} & & $D_{i j}^{c e q} a$ & $D_{i j}^{h} b$ & $D_{i j}^{\text {calc }}{ }_{c}$ & $D_{i j}^{o b s} d$ & $\Delta^{e}$ & $\operatorname{Corr}^{f}$ \\
\hline & $D_{12}$ & 39.191 & -0.1793 & 39.0112 & 39.1920 & -0.1808 & -0.0046 \\
\hline & $\mathbf{D}_{13}$ & 3.001 & -0.0056 & 2.9960 & 3.2730 & -0.2770 & -0.0017 \\
\hline & $D_{14}$ & -13.795 & 0.0732 & -13.7222 & -14.1050 & 0.3828 & -0.0052 \\
\hline & $\mathbf{D}_{15}$ & -42.847 & -0.0431 & -42.8858 & -42.9570 & 0.0712 & 0.0010 \\
\hline & $\mathbf{D}_{16}$ & -31.297 & -0.0385 & -31.3358 & -31.8300 & 0.4942 & 0.0012 \\
\hline & $\mathbf{D}_{\mathbf{1 7}}^{b}$ & 29.472 & -0.0790 & 29.3933 & 29.4330 & -0.0397 & -0.0027 \\
\hline & $\mathbf{D}_{18}^{b}$ & -1.977 & -0.0405 & -2.0173 & -1.4900 & -0.5273 & 0.0272 \\
\hline & $\mathbf{D}_{36}$ & 9.312 & -0.0407 & 9.2716 & 9.7710 & -0.4994 & -0.0042 \\
\hline & $\mathbf{D}_{37}$ & 32.373 & -0.2785 & 32.0942 & 31.4830 & 0.6112 & -0.0088 \\
\hline & $\mathbf{D}_{78}$ & -426.497 & 13.7956 & -412.7017 & -412.6470 & -0.0547 & -0.0334 \\
\hline & $\mathbf{R}$ & \multicolumn{6}{|c|}{0.40} \\
\hline
\end{tabular}

a partially averaged dipolar couplings values calculated for the molecule at its equilibrium state, ${ }^{b}$ vibrational motion contribution to be added to $D^{\text {ceq }}$ 's, ${ }^{c} D^{\text {calc }}=D^{c e q}+D^{h}$, ${ }^{d}$ partially averaged dipolar couplings values observed, ${ }^{e} \Delta=D^{\text {calc }}-D^{\text {obs }},{ }^{f} D^{h} / D^{\text {obs }}=$ Corr.

TABLE SI-3 : "Equilibrium", Calculated and Observed Dipolar Couplings; Harmonic Vibrational Corrections, Deviations, Relative Corrections and R for NBD at different temperatures in MM. Geometry and Force Field from B3LYP/6-31++G** calculations; experimental frequencies have been used in evaluating vibrational corrections (see text for more explanations). All dimensional values in $\mathrm{Hz}$.
$\mathbf{T}_{\text {red }}$
$D_{i j}^{c e q} a$
$D_{i j}^{h}$
$D_{i j}^{\text {calc } c}$
$D_{i j}^{o b s}{ }_{d}$
$\Delta^{e} \quad \operatorname{Corr}^{f}$ 
J. Phys. Chem. B, 2006, $x x x, x x x-x x x$

\begin{tabular}{|c|c|c|c|c|c|c|c|}
\hline \multirow{11}{*}{0.91} & $D_{12}$ & -117.252 & 1.1339 & -116.1184 & -116.6360 & 0.5176 & -0.0097 \\
\hline & $\mathbf{D}_{13}$ & -15.896 & 0.1128 & -15.7835 & -15.5950 & -0.1885 & -0.0072 \\
\hline & $D_{14}$ & -21.048 & 0.0579 & -20.9906 & -21.1120 & 0.1214 & -0.0027 \\
\hline & $\mathbf{D}_{15}$ & -37.377 & 0.0260 & -37.3509 & -37.6990 & 0.3481 & -0.0007 \\
\hline & $D_{16}$ & 15.876 & 0.2589 & 16.1347 & 15.6970 & 0.4377 & 0.0165 \\
\hline & $\mathbf{D}_{\mathbf{1 7}}^{b}$ & 68.917 & -0.2595 & 68.6574 & 68.9330 & -0.2756 & -0.0038 \\
\hline & $\mathbf{D}_{18}^{b}$ & 11.884 & -0.1326 & 11.7512 & 11.5930 & 0.1582 & -0.0114 \\
\hline & $\mathbf{D}_{36}$ & -27.861 & 0.1118 & -27.7491 & -26.9830 & -0.7661 & -0.0041 \\
\hline & $\mathbf{D}_{37}$ & -27.350 & -0.3869 & -27.7370 & -27.3820 & -0.3550 & 0.0141 \\
\hline & $\mathbf{D}_{78}$ & -372.086 & 12.4427 & -359.6432 & -359.3130 & -0.3302 & -0.0346 \\
\hline & $\mathbf{R}$ & \multicolumn{6}{|c|}{0.34} \\
\hline \multirow{12}{*}{0.92} & & $D_{i j}^{c e q} a$ & $D_{i j}^{h} b$ & $D_{i j}^{c a l c}{ }_{c}$ & $D_{i j}^{o b s} d$ & $\Delta^{e}$ & $\operatorname{Corr}^{f}$ \\
\hline & $\mathbf{D}_{12}$ & -109.061 & 1.0606 & -108.0005 & -108.5360 & 0.5355 & -0.0098 \\
\hline & $\mathbf{D}_{13}$ & -14.854 & 0.1059 & -14.7481 & -14.6450 & -0.1031 & -0.0072 \\
\hline & $D_{14}$ & -20.193 & 0.0566 & -20.1360 & -20.2740 & 0.1380 & -0.0028 \\
\hline & $\mathbf{D}_{15}$ & -36.398 & 0.0231 & -36.3750 & -36.8330 & 0.4580 & -0.0006 \\
\hline & $D_{16}$ & 14.000 & 0.2422 & 14.2422 & 13.8460 & 0.3962 & 0.0175 \\
\hline & $\mathbf{D}_{17}^{b}$ & 65.651 & -0.2463 & 65.4051 & 65.6120 & -0.2069 & -0.0038 \\
\hline & $\mathbf{D}_{18}^{b}$ & 11.112 & -0.1258 & 10.9866 & 10.7420 & 0.2446 & -0.0117 \\
\hline & $\mathbf{D}_{36}$ & -25.914 & 0.1039 & -25.8106 & -25.1190 & -0.6916 & -0.0041 \\
\hline & $\mathbf{D}_{37}$ & -24.754 & -0.3719 & -25.1259 & -24.7470 & -0.3789 & 0.0150 \\
\hline & $\mathbf{D}_{78}$ & -362.343 & 12.1031 & -350.2396 & -349.9160 & -0.3236 & -0.0346 \\
\hline & $\mathbf{R}$ & \multicolumn{6}{|c|}{0.34} \\
\hline \multirow{12}{*}{0.93} & & $D_{i j}^{c e q} a$ & $D_{i j}^{h} b$ & $D_{i j}^{c a l c}{ }_{c}$ & $D_{i j}^{o b s} d$ & $\Delta^{e}$ & $\operatorname{Corr}^{f}$ \\
\hline & $\mathbf{D}_{12}$ & -101.231 & 0.9897 & -100.2412 & -100.7890 & 0.5478 & -0.0098 \\
\hline & $\mathbf{D}_{13}$ & -13.849 & 0.0991 & -13.7500 & -13.6980 & -0.0520 & -0.0072 \\
\hline & $\mathrm{D}_{14}$ & -19.297 & 0.0550 & -19.2425 & -19.4180 & 0.1755 & -0.0028 \\
\hline & $\mathbf{D}_{15}$ & -35.258 & 0.0206 & -35.2378 & -35.5740 & 0.3362 & -0.0006 \\
\hline & $\mathbf{D}_{16}$ & 12.303 & 0.2261 & 12.5289 & 12.0800 & 0.4489 & 0.0187 \\
\hline & $\mathbf{D}_{17}^{b}$ & 62.336 & -0.2330 & 62.1030 & 62.1620 & -0.0590 & -0.0037 \\
\hline & $\mathbf{D}_{18}^{b}$ & 10.368 & -0.1191 & 10.2487 & 10.2120 & 0.0367 & -0.0117 \\
\hline & $\mathbf{D}_{36}$ & -24.054 & 0.0963 & -23.9576 & -23.1780 & -0.7796 & -0.0042 \\
\hline & $\mathbf{D}_{37}$ & -22.358 & -0.3561 & -22.7143 & -22.4180 & -0.2963 & 0.0159 \\
\hline & $\mathbf{D}_{78}$ & -350.996 & 11.7123 & -339.2840 & -339.0410 & -0.2430 & -0.0345 \\
\hline & $\mathbf{R}$ & \multicolumn{6}{|c|}{0.31} \\
\hline \multirow{12}{*}{0.94} & & $D_{i j}^{c e q}{ }_{a}$ & $D_{i j}^{h} b$ & $D_{i j}^{c a l c}{ }_{c}$ & $D_{i j}^{o b s}{ }_{d}$ & $\Delta^{e}$ & $\operatorname{Corr}^{f}$ \\
\hline & $\mathbf{D}_{12}$ & -93.767 & 0.9215 & -92.8451 & -93.3560 & 0.5109 & -0.0099 \\
\hline & $\mathbf{D}_{13}$ & -12.882 & 0.0926 & -12.7897 & -12.4880 & -0.3017 & -0.0074 \\
\hline & $D_{14}$ & -18.364 & 0.0531 & -18.3113 & -18.4810 & 0.1697 & -0.0029 \\
\hline & $\mathbf{D}_{15}$ & -33.960 & 0.0182 & -33.9415 & -34.3870 & 0.4455 & -0.0005 \\
\hline & $D_{16}$ & 10.784 & 0.2106 & 10.9950 & 10.5400 & 0.4550 & 0.0200 \\
\hline & $\mathbf{D}_{\mathbf{1 7}}^{b}$ & 58.974 & -0.2197 & 58.7546 & 58.8290 & -0.0744 & -0.0037 \\
\hline & $\mathbf{D}_{18}^{b}$ & 9.650 & -0.1123 & 9.5379 & 9.4330 & 0.1049 & -0.0119 \\
\hline & $\mathbf{D}_{36}$ & -22.280 & 0.0892 & -22.1912 & -21.5020 & -0.6892 & -0.0041 \\
\hline & $\mathbf{D}_{37}$ & -20.163 & -0.3394 & -20.5028 & -20.3340 & -0.1688 & 0.0167 \\
\hline & $\mathbf{D}_{78}$ & -338.068 & 11.2708 & -326.7975 & -326.5520 & -0.2455 & -0.0345 \\
\hline & $\mathbf{R}$ & \multicolumn{6}{|c|}{0.32} \\
\hline \multirow[t]{9}{*}{0.95} & & $D_{i j}^{c e q}{ }_{a}$ & $D_{i j}^{h} b$ & $D_{i j}^{\text {calc }} c$ & $D_{i j}^{o b s} d$ & $\Delta^{e}$ & $\operatorname{Corr}^{f}$ \\
\hline & $\mathbf{D}_{12}$ & -85.909 & 0.8488 & -85.0599 & -85.4580 & 0.3981 & -0.0099 \\
\hline & $\mathbf{D}_{13}$ & -11.856 & 0.0856 & -11.7705 & -11.6740 & -0.0965 & -0.0073 \\
\hline & $D_{14}$ & -17.306 & 0.0508 & -17.2548 & -17.2910 & 0.0362 & -0.0029 \\
\hline & $\mathbf{D}_{15}$ & -32.389 & 0.0159 & -32.3734 & -32.7120 & 0.3386 & -0.0005 \\
\hline & $D_{16}$ & 9.282 & 0.1940 & 9.4756 & 9.1090 & 0.3666 & 0.0213 \\
\hline & $\mathbf{D}_{17}^{b}$ & 55.242 & -0.2051 & 55.0373 & 55.1530 & -0.1157 & -0.0037 \\
\hline & $\mathbf{D}_{18}^{b}$ & 8.887 & -0.1048 & 8.7827 & 8.7600 & 0.0227 & -0.0120 \\
\hline & $\mathbf{D}_{36}$ & -20.413 & 0.0816 & -20.3316 & -19.7480 & -0.5836 & -0.0041 \\
\hline
\end{tabular}


J. Phys. Chem. B, 2006, $x x x, x x x-x x x$

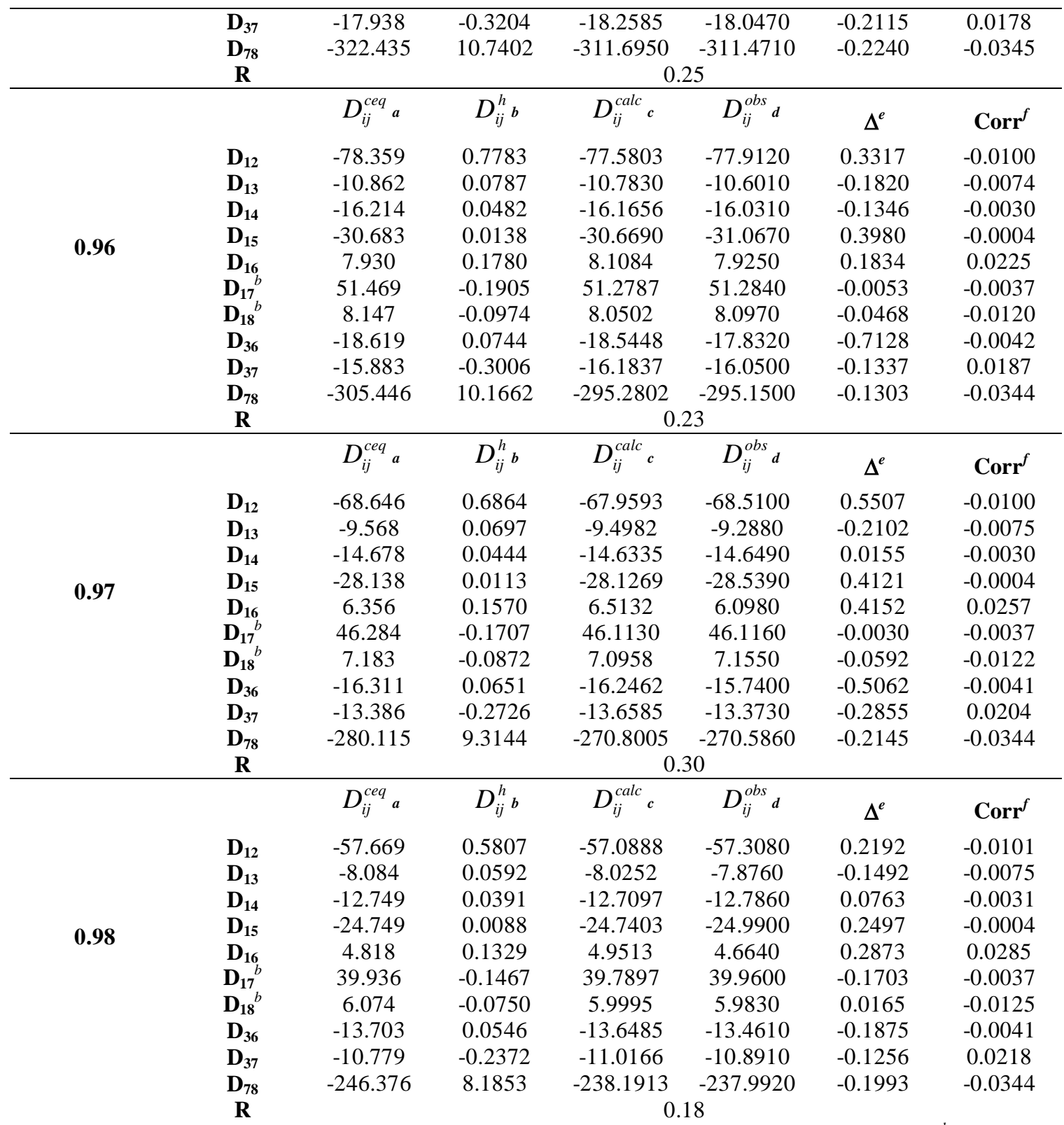

${ }^{a}$ partially averaged dipolar couplings values calculated for the molecule at its equilibrium state, ${ }^{b}$ vibrational motion contribution to be added to $D^{\text {ceq }}$ 's, ${ }^{c} D^{\text {calc }}=D^{\text {ceq }}+D^{h}$, ${ }^{d}$ partially averaged dipolar couplings values observed, ${ }^{e} \Delta=D^{\text {calc }}-D^{o b s},{ }^{f} D^{h} / D^{o b s}=$ Corr.

TABLE SI-4 : "Equilibrium", Calculated and Observed Dipolar Couplings; Harmonic Vibrational Corrections, Deviations, Relative Corrections and R for NBD at different temperatures in ZLI 1132. Geometry and Force Field from B3LYP/6-31++G** calculations; experimental frequencies have been used in evaluating vibrational corrections (see text for more explanations). All dimensional values in $\mathrm{Hz}$.

\begin{tabular}{|c|c|c|c|c|c|c|c|}
\hline $\mathbf{T}_{\text {red }}$ & & $D_{i j}^{c e q} a$ & $D_{i j b}^{h}$ & $D_{i j}^{\text {calc }}{ }_{c}$ & $D_{i j}^{o b s} d$ & $\Delta^{e}$ & Corr $^{f}$ \\
\hline \multirow[t]{7}{*}{0.90} & $\mathrm{D}_{12}$ & -310.589 & 2.7582 & -307.8310 & -309.1590 & 1.3280 & -0.0089 \\
\hline & $\mathrm{D}_{13}$ & -39.267 & 0.2593 & -39.0080 & -39.2070 & 0.1990 & -0.0066 \\
\hline & $D_{14}$ & -30.160 & 0.0397 & -30.1203 & -30.3220 & 0.2017 & -0.0013 \\
\hline & $\mathbf{D}_{15}$ & -31.015 & 0.1111 & -30.9038 & -30.3340 & -0.5698 & -0.0037 \\
\hline & $D_{16}$ & 73.989 & 0.6267 & 74.6160 & 74.1460 & 0.4700 & 0.0085 \\
\hline & $\mathbf{D}_{17}^{b}$ & 118.036 & -0.4837 & 117.5527 & 117.2100 & 0.3427 & -0.0041 \\
\hline & $\mathbf{D}_{18}{ }^{b}$ & 29.027 & -0.2470 & 28.7803 & 28.6500 & 0.1303 & -0.0086 \\
\hline
\end{tabular}


J. Phys. Chem. B, 2006, $x x x, x x x-x x x$

\begin{tabular}{|c|c|c|c|c|c|c|c|}
\hline & $\begin{array}{c}\mathbf{D}_{36} \\
\mathbf{D}_{37} \\
\mathbf{D}_{78} \\
\mathbf{R} \\
\end{array}$ & $\begin{array}{c}-73.801 \\
-100.992 \\
-308.753\end{array}$ & $\begin{array}{c}0.3002 \\
-0.5238 \\
10.8983\end{array}$ & $\begin{array}{r}-73.5004 \\
-101.5161 \\
-297.8550 \\
0 .\end{array}$ & $\begin{array}{c}-72.5650 \\
-100.4580 \\
-297.8190 \\
3\end{array}$ & $\begin{array}{l}-0.9354 \\
-1.0581 \\
-0.0360\end{array}$ & $\begin{array}{c}-0.0041 \\
0.0052 \\
-0.0366\end{array}$ \\
\hline 0.91 & $\begin{array}{l}\mathbf{D}_{12} \\
\mathbf{D}_{13} \\
\mathbf{D}_{14} \\
\mathbf{D}_{15} \\
\mathbf{D}_{16} \\
\mathbf{D}_{17}{ }^{b} \\
\mathbf{D}_{18}{ }^{b} \\
\mathbf{D}_{36} \\
\mathbf{D}_{37} \\
\mathbf{D}_{78}\end{array}$ & $\begin{array}{c}D_{i j}^{c e q} a \\
-292.214 \\
-37.012 \\
-28.991 \\
-30.816 \\
68.844 \\
112.606 \\
27.369 \\
-69.434 \\
-94.331 \\
-306.773\end{array}$ & $\begin{array}{c}D_{i j}^{h} \boldsymbol{b} \\
2.6010 \\
0.2449 \\
0.0401 \\
0.1035 \\
0.5911 \\
-0.4600 \\
-0.2349 \\
0.2823 \\
-0.5049 \\
10.7843\end{array}$ & $\begin{array}{c}D_{i j}^{\text {calc }}{ }^{2} \\
-289.6135 \\
-36.7676 \\
-28.9515 \\
-30.7125 \\
69.4347 \\
112.1456 \\
27.1341 \\
-69.1522 \\
-94.8356 \\
-295.9888\end{array}$ & $\begin{array}{c}D_{i j}^{o b s}{ }_{d} \\
-290.8620 \\
-36.8240 \\
-29.2010 \\
-30.0330 \\
68.8510 \\
112.3290 \\
26.4190 \\
-68.1590 \\
-93.7610 \\
-295.7510\end{array}$ & $\begin{array}{c}\Delta^{e} \\
1.2485 \\
0.0564 \\
0.2496 \\
-0.6795 \\
0.5837 \\
-0.1834 \\
0.7151 \\
-0.9932 \\
-1.0746 \\
-0.2378\end{array}$ & $\begin{array}{c}\text { Corr }^{f} \\
-0.0089 \\
-0.0067 \\
-0.0014 \\
-0.0034 \\
0.0086 \\
-0.0041 \\
-0.0089 \\
-0.0041 \\
0.0054 \\
-0.0365\end{array}$ \\
\hline 0.92 & $\begin{array}{c}\mathbf{D}_{12} \\
\mathbf{D}_{13} \\
\mathbf{D}_{14} \\
\mathbf{D}_{15} \\
\mathbf{D}_{16} \\
\mathbf{D}_{17}{ }^{b} \\
\mathbf{D}_{18}{ }^{b} \\
\mathbf{D}_{36} \\
\mathbf{D}_{37} \\
\mathbf{D}_{78} \\
\mathbf{R}\end{array}$ & $\begin{array}{c}D_{i j}^{c e q} a \\
-266.201 \\
-33.774 \\
-26.916 \\
-29.415 \\
62.085 \\
103.855 \\
24.981 \\
-63.253 \\
-85.370 \\
-292.822\end{array}$ & $\begin{array}{c}D_{i j \boldsymbol{b}}^{h} \\
2.3743 \\
0.2239 \\
0.0387 \\
0.0935 \\
0.5396 \\
-0.4231 \\
-0.2160 \\
0.2571 \\
-0.4698 \\
10.2597\end{array}$ & $\begin{array}{c}0 . \\
D_{i j}^{\text {calc }}{ }^{c} \\
-263.8271 \\
-33.5498 \\
-26.8771 \\
-29.3212 \\
62.6245 \\
103.4316 \\
24.7649 \\
-62.9963 \\
-85.8398 \\
-282.5626 \\
1 .\end{array}$ & \begin{tabular}{l}
9 \\
\multicolumn{1}{c}{$D_{i j}^{o b s}{ }_{d}$} \\
-266.0820 \\
-33.7670 \\
-27.1090 \\
-28.9370 \\
62.3690 \\
100.6710 \\
24.6610 \\
-62.2900 \\
-85.1820 \\
-283.5460 \\
6
\end{tabular} & $\begin{array}{c}\Delta^{e} \\
2.2549 \\
0.2172 \\
0.2319 \\
-0.3842 \\
0.2555 \\
2.7606 \\
0.1039 \\
-0.7063 \\
-0.6578 \\
0.9834\end{array}$ & $\begin{array}{c}\text { Corr }^{f} \\
-0.0089 \\
-0.0066 \\
-0.0014 \\
-0.0032 \\
0.0087 \\
-0.0042 \\
-0.0088 \\
-0.0041 \\
0.0055 \\
-0.0362\end{array}$ \\
\hline 0.93 & $\begin{array}{c}\mathbf{D}_{12} \\
\mathbf{D}_{13} \\
\mathbf{D}_{14} \\
\mathbf{D}_{15} \\
\mathbf{D}_{16} \\
\mathbf{D}_{17}{ }^{b} \\
\mathbf{D}_{18}{ }^{b} \\
\mathbf{D}_{36} \\
\mathbf{D}_{37} \\
\mathbf{D}_{78} \\
\mathbf{R} \\
\end{array}$ & $\begin{array}{c}D_{i j}^{c e q} a \\
-251.072 \\
-31.908 \\
-25.875 \\
-29.042 \\
57.946 \\
99.185 \\
23.608 \\
-59.658 \\
-79.972 \\
-289.114\end{array}$ & $\begin{array}{c}D_{i j \boldsymbol{b}}^{h} \\
2.2440 \\
0.2119 \\
0.0387 \\
0.0874 \\
0.5101 \\
-0.4029 \\
-0.2057 \\
0.2424 \\
-0.4527 \\
10.0982\end{array}$ & $\begin{array}{c}D_{i j}^{\text {calc }}{ }^{c} \\
-248.8279 \\
-31.6966 \\
-25.8365 \\
-28.9548 \\
58.4561 \\
98.7821 \\
23.4023 \\
-59.4160 \\
-80.4252 \\
-279.0160 \\
0 .\end{array}$ & $\begin{array}{c}D_{i j}{ }^{o b s}{ }_{d} \\
-249.9930 \\
-31.8100 \\
-26.0350 \\
-28.4520 \\
57.9430 \\
98.6870 \\
23.0870 \\
-58.6190 \\
-79.4800 \\
-278.8840 \\
7\end{array}$ & $\begin{array}{c}\Delta^{e} \\
1.1651 \\
0.1134 \\
0.1985 \\
-0.5028 \\
0.5131 \\
0.0951 \\
0.3153 \\
-0.7970 \\
-0.9452 \\
-0.1320\end{array}$ & $\begin{array}{c}\text { Corr }^{f} \\
-0.0090 \\
-0.0067 \\
-0.0015 \\
-0.0031 \\
0.0088 \\
-0.0041 \\
-0.0089 \\
-0.0041 \\
0.0057 \\
-0.0362\end{array}$ \\
\hline 0.94 & $\begin{array}{c}\mathbf{D}_{12} \\
\mathbf{D}_{13} \\
\mathbf{D}_{14} \\
\mathbf{D}_{15} \\
\mathbf{D}_{16} \\
\mathbf{D}_{17}{ }^{b} \\
\mathbf{D}_{18}{ }^{b} \\
\mathbf{D}_{36} \\
\mathbf{D}_{37} \\
\mathbf{D}_{78} \\
\mathbf{R}\end{array}$ & $\begin{array}{c}D_{i j}^{c e q} a \\
-235.661 \\
-29.999 \\
-24.726 \\
-28.425 \\
53.842 \\
94.203 \\
22.201 \\
-55.997 \\
-74.574 \\
-282.974\end{array}$ & $\begin{array}{c}D_{i j}^{h} \boldsymbol{b} \\
2.1105 \\
0.1996 \\
0.0383 \\
0.0813 \\
0.4798 \\
-0.3817 \\
-0.1949 \\
0.2275 \\
-0.4335 \\
9.8566\end{array}$ & $\begin{array}{c}D_{i j}^{\text {calc }}{ }_{c} \\
-233.5510 \\
-29.7991 \\
-24.6875 \\
-28.3441 \\
54.3216 \\
93.8217 \\
22.0061 \\
-55.7692 \\
-75.0080 \\
-273.1174 \\
0 .\end{array}$ & $\begin{array}{c}D_{i j}^{o b s}{ }_{d} \\
-234.6970 \\
-29.9250 \\
-24.8440 \\
-27.9300 \\
53.8030 \\
93.6700 \\
21.8900 \\
-54.9570 \\
-74.0900 \\
-272.9930 \\
5\end{array}$ & $\begin{array}{c}\Delta^{e} \\
1.1460 \\
0.1259 \\
0.1565 \\
-0.4141 \\
0.5186 \\
0.1517 \\
0.1161 \\
-0.8122 \\
-0.9180 \\
-0.1244\end{array}$ & $\begin{array}{c}\text { Corr }^{f} \\
-0.0090 \\
-0.0067 \\
-0.0015 \\
-0.0029 \\
0.0089 \\
-0.0041 \\
-0.0089 \\
-0.0041 \\
0.0059 \\
-0.0361\end{array}$ \\
\hline 0.95 & $\begin{array}{l}D_{12} \\
D_{13}\end{array}$ & $\begin{array}{c}D_{i j}^{c e q} a \\
-219.281 \\
-27.962\end{array}$ & $\begin{array}{c}D_{i j \boldsymbol{b}}^{h} \\
1.9680 \\
0.1864 \\
\end{array}$ & $\begin{array}{c}D_{i j}^{\text {calc }}{ }^{c} \\
-217.3130 \\
-27.7755\end{array}$ & $\begin{array}{c}D_{i j}^{o b s} d \\
-218.3760 \\
-27.8640\end{array}$ & $\begin{array}{c}\Delta^{e} \\
1.0630 \\
0.0885 \\
\end{array}$ & $\begin{array}{c}\text { Corr }^{f} \\
-0.0090 \\
-0.0067 \\
\end{array}$ \\
\hline
\end{tabular}


J. Phys. Chem. B, 2006, $x x x, x x x-x x x$

\begin{tabular}{|c|c|c|c|c|c|c|c|}
\hline & $\mathrm{D}_{14}$ & -23.443 & 0.0376 & -23.4052 & -23.5370 & 0.1318 & -0.0016 \\
\hline & $\mathbf{D}_{15}$ & -27.607 & 0.0749 & -27.5319 & -27.2100 & -0.3219 & -0.0028 \\
\hline & $D_{16}$ & 49.556 & 0.4474 & 50.0032 & 49.4960 & 0.5072 & 0.0090 \\
\hline & $\mathbf{D}_{17}^{b}$ & 88.753 & -0.3586 & 88.3949 & 88.2720 & 0.1229 & -0.0041 \\
\hline & $\mathbf{D}_{18}^{b}$ & 20.700 & -0.1831 & 20.5165 & 20.3820 & 0.1345 & -0.0090 \\
\hline & $\mathbf{D}_{36}$ & -52.104 & 0.2116 & -51.8928 & -51.0580 & -0.8348 & -0.0041 \\
\hline & $\mathbf{D}_{37}$ & -68.905 & -0.4119 & -69.3170 & -68.5020 & -0.8150 & 0.0060 \\
\hline & $\mathbf{D}_{78}$ & -274.825 & 9.5470 & -265.2782 & -265.1460 & -0.1322 & -0.0360 \\
\hline & $\mathbf{R}$ & \multicolumn{6}{|c|}{0.50} \\
\hline \multirow{12}{*}{0.96} & & $D_{i j}^{c e q} a$ & $D_{i j}^{h} b$ & $D_{i j}^{c a l c}{ }_{c}$ & $D_{i j}^{o b s}{ }_{d}$ & $\Delta^{e}$ & $\operatorname{Corr}^{f}$ \\
\hline & $\mathbf{D}_{12}$ & -201.089 & 1.8088 & -199.2801 & -200.3060 & 1.0259 & -0.0090 \\
\hline & $\mathbf{D}_{13}$ & -25.689 & 0.1716 & -25.5177 & -25.5150 & -0.0027 & -0.0067 \\
\hline & $D_{14}$ & -21.923 & 0.0363 & -21.8872 & -22.0310 & 0.1438 & -0.0016 \\
\hline & $\mathrm{D}_{15}$ & -26.447 & 0.0680 & -26.3790 & -26.0960 & -0.2830 & -0.0026 \\
\hline & $D_{16}$ & 44.913 & 0.4113 & 45.3248 & 44.8070 & 0.5178 & 0.0092 \\
\hline & $\mathbf{D}_{17}^{b}$ & 82.463 & -0.3323 & 82.1308 & 82.0230 & 0.1078 & -0.0041 \\
\hline & $\mathbf{D}_{18}^{b}$ & 19.023 & -0.1697 & 18.8534 & 18.6030 & 0.2504 & -0.0091 \\
\hline & $\mathbf{D}_{36}$ & -47.782 & 0.1940 & -47.5877 & -46.9270 & -0.6607 & -0.0041 \\
\hline & $\mathbf{D}_{37}$ & -62.714 & -0.3860 & -63.1000 & -62.3610 & -0.7390 & 0.0062 \\
\hline & $\mathbf{D}_{78}$ & -263.279 & 9.1217 & -254.1571 & -254.0240 & -0.1331 & -0.0359 \\
\hline & $\mathbf{R}$ & \multicolumn{6}{|c|}{0.47} \\
\hline \multirow{12}{*}{0.97} & & $D_{i j}^{c e q} a$ & $D_{i j}^{h} b$ & $D_{i j}^{c a l c}{ }_{c}$ & $D_{i j}^{o b s}{ }_{d}$ & $\Delta^{e}$ & $\operatorname{Corr}^{f}$ \\
\hline & $D_{12}$ & -181.837 & 1.6394 & -180.1973 & -181.1180 & 0.9207 & -0.0091 \\
\hline & $\mathbf{D}_{13}$ & -23.274 & 0.1558 & -23.1184 & -23.2290 & 0.1106 & -0.0067 \\
\hline & $D_{14}$ & -20.224 & 0.0346 & -20.1898 & -20.2890 & 0.0992 & -0.0017 \\
\hline & $D_{15}$ & -24.977 & 0.0608 & -24.9162 & -24.6570 & -0.2593 & -0.0025 \\
\hline & $D_{16}$ & 40.115 & 0.3728 & 40.4875 & 39.9960 & 0.4915 & 0.0093 \\
\hline & $\mathbf{D}_{17}^{b}$ & 75.576 & -0.3036 & 75.2723 & 75.1760 & 0.0963 & -0.0040 \\
\hline & $\mathbf{D}_{18}^{b}$ & 17.240 & -0.1550 & 17.0851 & 16.9460 & 0.1391 & -0.0091 \\
\hline & $\mathbf{D}_{36}$ & -43.207 & 0.1753 & -43.0318 & -42.3850 & -0.6468 & -0.0041 \\
\hline & $\mathbf{D}_{37}$ & -56.264 & -0.3569 & -56.6208 & -55.9310 & -0.6898 & 0.0064 \\
\hline & $\mathbf{D}_{78}$ & -248.646 & 8.5930 & -240.0531 & -239.9240 & -0.1291 & -0.0358 \\
\hline & $\mathbf{R}$ & \multicolumn{6}{|c|}{0.43} \\
\hline
\end{tabular}

apartially averaged dipolar couplings values calculated for the molecule at its equilibrium state, ${ }^{b}$ vibrational motion contribution to be added to $D^{\text {ceq }}$ 's, ${ }^{c} D^{\text {calc }}=D^{c e q}+D^{h}$, ${ }^{d}$ partially averaged dipolar couplings values observed, ${ }^{e} \Delta=D^{\text {calc }}-D^{\text {obs }},{ }^{f} D^{h} / D^{\text {obs }}=$ Corr.

TABLE SI-5 : Experimental and MC-calculated $S_{z z}$ and $S_{x x}-S_{y y}$ values (estimated error on experimental values: $\pm 1 \%$; simulated points within a $5 \%$ of statistical uncertainty, due to "finiteness" of random sampling process).

\begin{tabular}{|c|c|c|c|c|c|c|c|c|c|c|c|c|}
\hline \multirow[b]{3}{*}{$\mathbf{T}_{\text {red }}$} & \multicolumn{4}{|c|}{ EBBA } & \multicolumn{4}{|c|}{ MM } & \multicolumn{4}{|c|}{ ZLI 1132} \\
\hline & \multicolumn{2}{|c|}{$\mathbf{S}_{\mathbf{z z}}$} & \multicolumn{2}{|c|}{$S_{x x}-S_{y y}$} & \multicolumn{2}{|c|}{$\mathbf{S}_{\mathbf{z z}}$} & \multicolumn{2}{|c|}{$S_{x x}-S_{y y}$} & \multicolumn{2}{|c|}{$\mathbf{S}_{\mathbf{z z}}$} & \multicolumn{2}{|c|}{$S_{x x}-S_{y y}$} \\
\hline & $\begin{array}{c}\text { experimen } \\
\text { tal }\end{array}$ & $\begin{array}{c}M C- \\
\text { calculated }\end{array}$ & $\begin{array}{c}\text { experimen } \\
\text { tal }\end{array}$ & $\begin{array}{c}M C- \\
\text { calculated }\end{array}$ & $\begin{array}{l}\text { experimen } \\
\text { tal }\end{array}$ & $\begin{array}{c}M C- \\
\text { calculated }\end{array}$ & $\begin{array}{c}\text { experimen } \\
\text { tal }\end{array}$ & $\begin{array}{c}M C- \\
\text { calculated }\end{array}$ & $\begin{array}{l}\text { experimen } \\
\text { tal }\end{array}$ & $\begin{array}{c}M C- \\
\text { calculated }\end{array}$ & $\begin{array}{c}\text { experimen } \\
\text { tal }\end{array}$ & $\begin{array}{c}M C- \\
\text { calculated }\end{array}$ \\
\hline 0.90 & & & & & & -0.0384 & & 0.0001 & -0.0650 & -0.0644 & -0.0346 & -0.0327 \\
\hline 0.91 & -0.0209 & -0.0231 & 0.0409 & 0.0353 & -0.0368 & & -0.0007 & & -0.0616 & -0.0595 & -0.0318 & -0.0290 \\
\hline 0.92 & -0.0203 & & 0.0394 & & -0.0350 & -0.0355 & 0.0002 & 0.0016 & -0.0567 & & -0.0283 & \\
\hline 0.93 & -0.0197 & & 0.0379 & & -0.0332 & & 0.0009 & & -0.0541 & -0.0556 & -0.0260 & -0.0270 \\
\hline 0.94 & -0.0190 & -0.0201 & 0.0363 & 0.0332 & -0.0314 & -0.0317 & 0.0014 & 0.0014 & -0.0514 & -0.0502 & -0.0239 & -0.0261 \\
\hline 0.95 & -0.0181 & & 0.0344 & & -0.0294 & & 0.0019 & & -0.0484 & & -0.0217 & \\
\hline
\end{tabular}


J. Phys. Chem. B, 2006, $x x x, x x x-x x x$

\begin{tabular}{|l|l|l|l|l|l|l|l|l|l|l|l|l|}
$\mathbf{0 . 9 6}$ & -0.0172 & & 0.0324 & & -0.0274 & -0.0264 & 0.0023 & -0.0002 & -0.0449 & -0.0453 & -0.0193 & -0.0205 \\
$\mathbf{0 . 9 7}$ & -0.0161 & -0.0159 & 0.0300 & 0.0293 & -0.0246 & & 0.0026 & & -0.0411 & -0.0405 & -0.0170 & -0.0209 \\
$\mathbf{0 . 9 8}$ & -0.0144 & & 0.0267 & & -0.0212 & -0.0219 & 0.0027 & -0.0008 & & & & \\
\hline
\end{tabular}

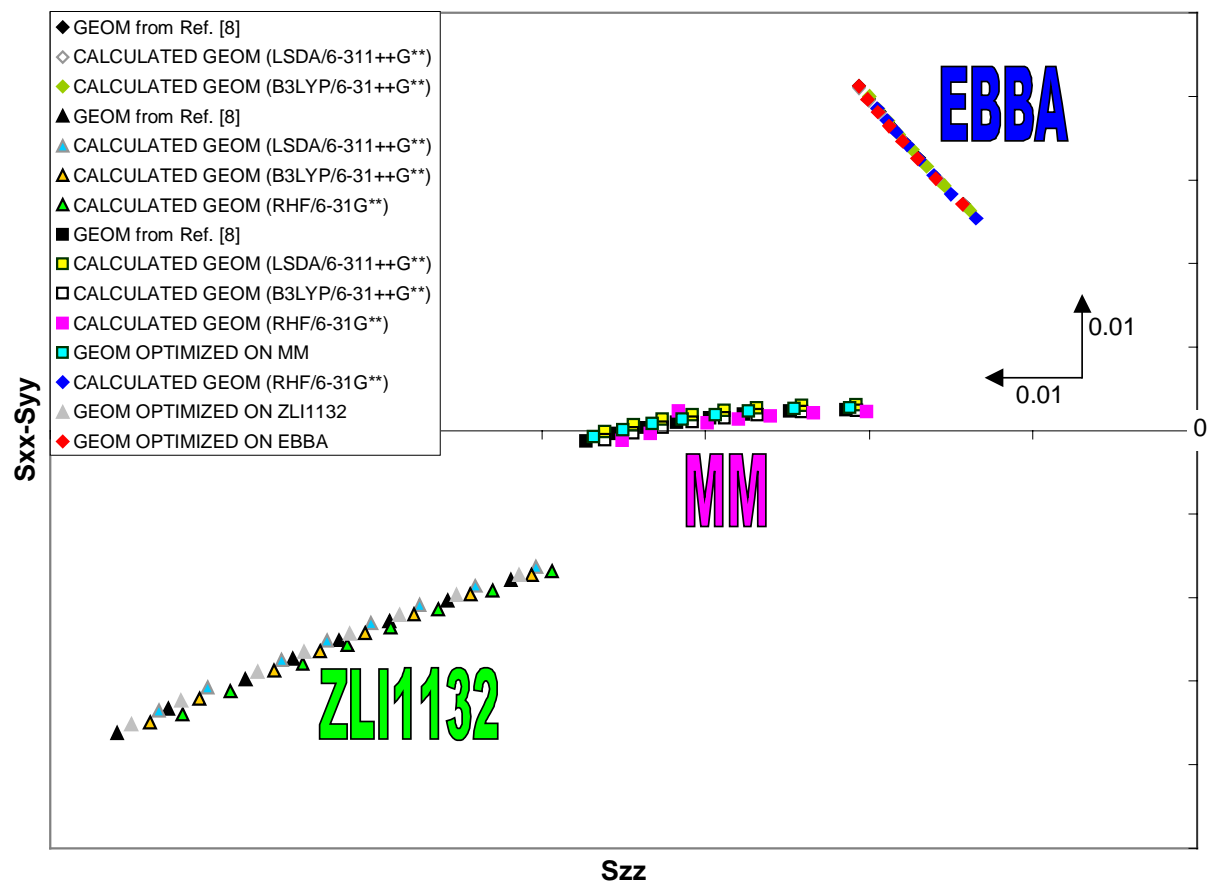

Figure SI-1: "Scattering” of order parameters corresponding to different choices of geometries.

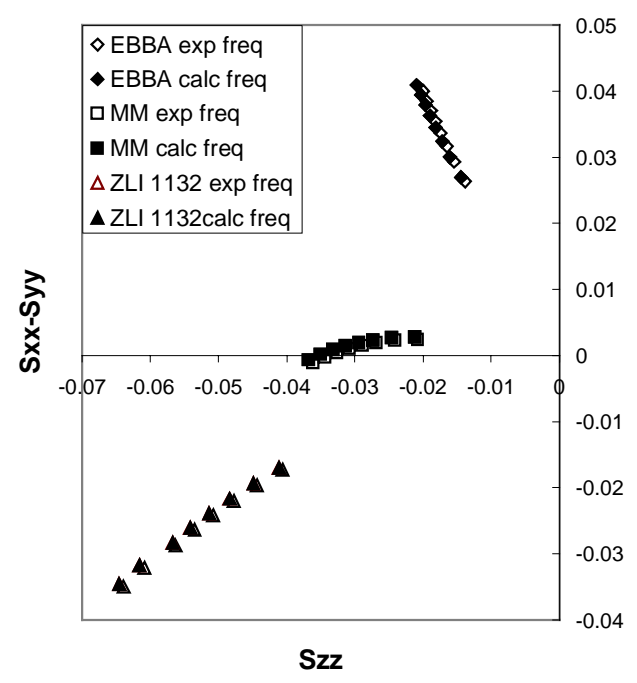

Figure SI-2: Effect on order parameters of using experimental or calculated frequencies in the vibrational corrections (the data shown in figure are obtained by using geometries and Force Field from B3LYP/6$31++G * *)$. 